\title{
The anatomical variations of the lateral sesamoid bones of the foot: a retrospective radiographic analysis
}

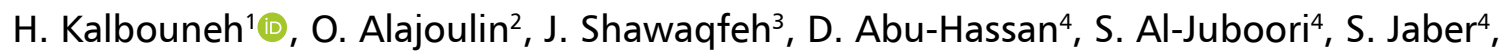 \\ L. Alshabatat ${ }^{4}$, T. Massad ${ }^{4}$, M. Alsalem ${ }^{1}$ \\ ${ }^{1}$ Department of Anatomy, Faculty of Medicine, The University of Jordan, Amman, Jordan \\ ${ }^{2}$ Orthopedic and Trauma Department, Ankle and Foot Section, Jordanian Royal Medical Services, Jordan \\ ${ }^{3}$ Radiology Department, Jordanian Royal Medical Services, Jordan \\ ${ }^{4}$ Faculty of Medicine, The University of Jordan, Amman, Jordan
}

[Received: 30 August 2021; Accepted: 21 September 2021; Early publication date: 7 October 2021]

\begin{abstract}
Background: Os peroneum and os vesalianum are sesamoid bones that could be found within fibularis longus and brevis tendons, respectively. They are rarely a cause of lateral foot pain and are often identified as incidental radiographic findings. However, in the context of trauma, these sesamoids may be radiographically misinterpreted as fractures. This study aimed to evaluate the prevalence and normal morphological variants of os peroneum and os vesalianum.

Materials and methods: Standard oblique lateral and/or anteroposterior radiographic views of 624 feet of adolescent and adult patients were retrospectively reviewed to determine the prevalence and anatomical variations of the os peroneum and os vesalianum in relation to age and gender using plain radiography. Results: Os peroneum was found in $22 \%$ and os vesalianum was found in $1.6 \%$. Age was found to significantly correlate with the presence of os peroneum with the highest prevalence (30\%) detected in the elderly group. Among 137 feet with os peroneum, $54.0 \%$ were between 4 and $8 \mathrm{~mm}, 67.2 \%$ were close to the tubercle of cuboid, 32.8\% were located at the level of calcaneocuboid joint, $81.8 \%$ were solitary, and $18.2 \%$ were bi-/multipartite. Among 10 feet with os vesalianum, type / was identified in 40\% and type // in 60\%.

Conclusions: Different anatomical variants of the lateral sesamoid bones of the foot have been described in this study. A thorough knowledge of normal anatomical variants is essential for proper diagnosis and management and can enhance our diagnostic skills in detecting these sesamoids. (Folia Morphol 2022; 81, 4: 983-990)
\end{abstract}

Key words: os peroneum, os vesalianum, prevalence, sesamoids, variants, morphology

\section{INTRODUCTION}

Sesamoid bones and accessory ossicles of the foot are osseous structures that, despite being asymptomatic in most individuals, are increasingly being reported in the literature due to the pain directly related to them or the misinterpretation of them as fractures [18, 21]. Sesamoid bones are located within tendons as they pass over joints to protect 
tendons against friction $[8,16]$. Accessory ossicles are, unlike sesamoids, structures of unknown function; they originate from ossification centres that have failed to fuse with the main bone $[8,16]$. Although different, sesamoid bones and accessory ossicles do share similar imaging characteristics; both are small structures that could be ovoid or nodular, unilateral or bilateral, and may be present near a bone or a site of articulation [21].

Os peroneum is a round or oval sesamoid bone embedded within the fibularis (peroneus) longus tendon. It is related to the plantar or lateral surface of cuboid. There is controversy about the origin of os peroneum. It has been postulated to be developed as a result of mechanical stresses imposed on the fibularis longus tendon at the point where the tendon turns medially [19]. Guimera et al. [13] however, showed that os peroneum is already present during embryonic development period. It was reported by many authors that the fibularis longus tendon is interrupted by the presence of a fibrocartilagenous node at the point of articulation with the tubercle of cuboid $[3,4,15,20,24]$. Histologically, the fibrocartilage of fibularis longus tendon showed different degrees of osseous, cartilaginous and fibrous tissue, so it may or may not display the presence of an os peroneum [20]. The prevalence of the cartilaginous form of os peroneum remains unclear [28]. However, its ossified form is a relatively common anatomical variant; seen in up to $32 \%$ of feet $[9,20]$. Os peroneum is bipartite in approximately $30 \%$ of cases and bilateral in approximately $60 \%$ [5].

Os vesalianum is located near the base of the fifth metatarsal bone and is found within the fibularis brevis tendon at its insertion [21]. Radiographically, os vesalianum is separated from the fifth metatarsal base by a thin, radiolucent line that may represent a synchondrosis [7]. The radiographic prevalence has been estimated from $0.1 \%$ and $5.9 \%[9,10,26]$.

Despite meeting the criteria of sesamoid bones, os peroneum and os vesalianum have been consistently classified throughout the literature as accessory ossicles $[8,21]$. However, they were described in this manuscript as sesamoid bones. Typically, sesamoid bones are considered asymptomatic and are rarely associated with pathological findings such as fractures or degenerative changes in response to overuse and trauma. Although no further diagnostic workup is required, it is essential to recognise these sesamoids and the possibility of symptoms directly related to their presence. A solid understanding of anatomy is fundamental in understanding pathologies that cause symptoms in the lateral aspect of the foot [17]. Both sesamoids and accessory ossicles are subjected to significant morphological variations. There are insufficient studies reported in the orthopaedic and anatomic literature regarding the anatomic variations and configuration of the lateral sesamoid bones of the foot. The aim of this study was to evaluate the relative frequency of occurrence of the lateral sesamoid bones of the foot according to age and gender using plain radiography and to assess the different anatomical variants with their characteristic imaging findings.

\section{MATERIALS AND METHODS}

Standard oblique lateral and/or anteroposterior radiographic views of the feet of adolescent and adult subjects were retrospectively reviewed. The inclusion criteria were patients aged 14 years and older. All patients with prior foot surgeries and pathologies including osteolysis, severe arthritis, and calcification were excluded. Midfoot fractures were also excluded. The Institutional Review Board of Jordanian Royal Medical Services and the University of Jordan approved the study and waived the requirement for informed consent due to the retrospective nature of this study.

Radiographic views of 624 patients (346 men and 278 women) with 302 left and 322 right feet were included. The presence of lateral foot sesamoid bones (os peroneum and os vesalianum) was evaluated by three researchers independently. Two anatomical variants for os vesalianum were distinguished (Fig. 1): Type I: a small os vesalianum, 2 to $4 \mathrm{~mm}$ in size, and adjacent to the tip of the well-developed tuberosity of the fifth metatarsal bone and type II: a large os vesalianum, 10 to $20 \mathrm{~mm}$ in size, and oblong in shape with well-formed cortical margins and a clear separation from the base of the fifth metatarsal and cuboid by a radiolucent line of uniform width.

Regarding os peroneum, different anatomical variants were identified depending on size (from its largest dimension on the radiograph), location relative to calcaneocuboid joint (assessed in the oblique lateral radiographs), and radiographic appearance (solitary, bipartite or multipartite). Three groups of os peroneum were distinguished based on the maximum measured diameter: smaller than $4 \mathrm{~mm}$, between 4 and $8 \mathrm{~mm}$, and larger than $8 \mathrm{~mm}$. According to its location assessed in the oblique lateral radiographs, 


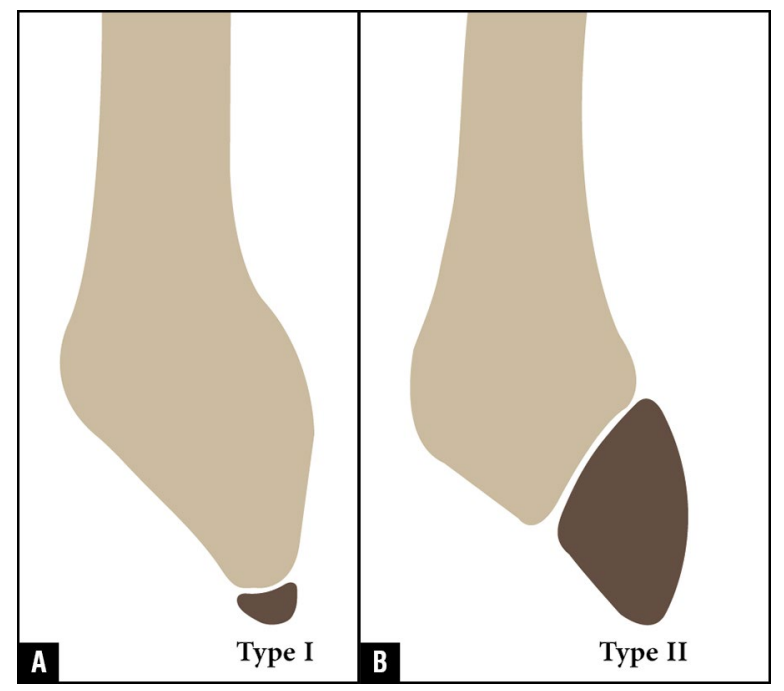

Figure 1. Schematic illustration of os vesalianum types according to their radiographic appearance; A. Type I os vesalianum; B. Type II os vesalianum.

two positions were distinguished: in close relationship to the tubercle of cuboid distal to calcaneocuboid joint or at the level of calcaneocuboid joint. Individual age and gender were reviewed from hospital records. The subjects were divided into four separate age groups: teenagers (14-19 years), young adults ( $20-39$ years), middle-aged ( $40-59$ years) and elderly (60 years and over).

\section{Statistical analysis}

GraphPad Prism version 6.04 for Windows (GraphPad Software, La Jolla, CA) was used. The prevalence of os peroneum and os vesalianum was compared between genders (male vs. female) and sides (right vs. left) using Fisher's exact test. Chi-squared test was used for comparisons between different age groups. The point-biserial correlation coefficient $\left(r_{p b}\right)$ was used to measure the strength of association between age and the lateral sesamoid bones (dichotomous variable). Inter-rater reliability was analysed using Cohen's kappa. The significance threshold was set at 0.05 .

\section{RESULTS}

The average age was 36.2 (range: 14-82) years. Of the subjects, $55.4 \%$ were males. The prevalence of os peroneum and os vesalianum in number and percentage according to gender and side is shown in Table 1. Lateral sesamoid bones of the foot were found in $23.6 \%$ (147/624). Os peroneum was identified in $22.0 \%(137 / 624)$ and os vesalianum was identified in $1.6 \%$ (10/624). No significant differences in the prevalence of lateral foot sesamoid bones were found between genders and sides $(p=0.06$ and 0.71 , respectively) (Table 1).

The prevalence of os peroneum and os vesalianum in number and percentage in different age groups is shown in Table 2 . A statistically significant difference was found in the prevalence of os peroneum between different age groups $(p=0.04)$. Os peroneum was found in $14.6 \%$ (15/103) of adolescents, $20.4 \%$ (60/294) of young adults, $25.6 \%(43 / 168)$ of middle-aged adults and in $32.2 \%$ (19/59) of the elderly group ( $\geq 60$ years). A statistically significant moderate positive correlation was found between age and os peroneum ( $r_{p b}=0.31, p<0.001$ ) (Fig. 2A).

No statistically significant difference was found in the prevalence of os vesalianum between different age groups $(p=0.96)$. Os vesalianum was found in $1.9 \%(2 / 103)$ of adolescents, $1.7 \%$ (5/294) of young adults, $1.2 \%(2 / 168)$ of middle-aged adults, and in $1.6 \%(1 / 59)$ of the elderly group. A weak negative correlation was found between age and os vesalianum ( $r_{p b}=-0.04, p=0.35$ ) (Fig. 2B).

The different anatomical variants of the lateral foot sesamoid bones were shown in Table 3. Among 137 feet with os peroneum, 29.2\% (40/137) were

Table 1. Prevalence of os peroneum and vesalianum according to gender and side

\begin{tabular}{lccccc}
\hline & All $\mathbf{( n = 6 2 4 )}$ & Male $(\mathbf{n}=\mathbf{3 4 6})$ & Female $(\mathbf{n}=\mathbf{2 7 8})$ & Right $(\mathbf{n}=\mathbf{3 2 2})$ & Left $(\mathbf{n}=\mathbf{3 0 2})$ \\
\hline Lateral ossicles & $147(23.6 \%)$ & $92(26.6 \%)$ & $55(19.8 \%)$ & $78(24.2 \%)$ & $69(22.8 \%)$ \\
P & & $0.06^{\mathrm{NS}}$ & & $0.70^{\mathrm{NS}}$ & \\
Os peroneum & $137(22.0 \%)$ & $85(24.6 \%)$ & $52(18.7 \%)$ & $72(22.4 \%)$ & $65(21.5 \%)$ \\
P & & $0.08^{\mathrm{NS}}$ & & $0.85^{\mathrm{NS}}$ & \\
Os vesalianum & $10(1.6 \%)$ & $7(2.0 \%)$ & $3(1.1 \%)$ & $6(1.9 \%)$ & $4(1.3 \%)$ \\
P & & $0.52^{\mathrm{NS}}$ & & $0.75^{\mathrm{NS}}$ &
\end{tabular}

NS - not significant at $p<0.05$. Fisher Exact Test 
Table 2. Prevalence of os peroneum and os vesalianum according to age

\begin{tabular}{lcccc}
\hline & \multicolumn{4}{c}{ Age groups [years] } \\
\cline { 2 - 5 } & $\mathbf{1 4 - 1 9}$ & $\mathbf{2 0 - 3 9}$ & $\mathbf{4 0 - 5 9}$ & $\geq \mathbf{6 0}$ \\
\hline Overall $(\mathrm{n}=624)$ & $103(16.5 \%)$ & $294(47.1 \%)$ & $168(26.9 \%)$ & $59(9.5 \%)$ \\
Os peroneum $(\mathrm{n}=137)$ & $15(14.6 \%)$ & $60(20.4 \%)$ & $43(25.6 \%)$ & $19(32.2 \%)$ \\
Chi-squared & & & 8.611 & \\
P value & & & $0.04^{*}$ & $1(1.6 \%)$ \\
Os vesalianum $(\mathrm{n}=10)$ & $2(1.9 \%)$ & $5(1.7 \%)$ & $2(1.2 \%)$ & \\
Chi-squared & & & 0.277 & \\
P value & & & $0.96^{\mathrm{NS}}$ & \\
\hline
\end{tabular}

NS - not significant; ${ }^{*}$ significant $\mathrm{p}<0.05$
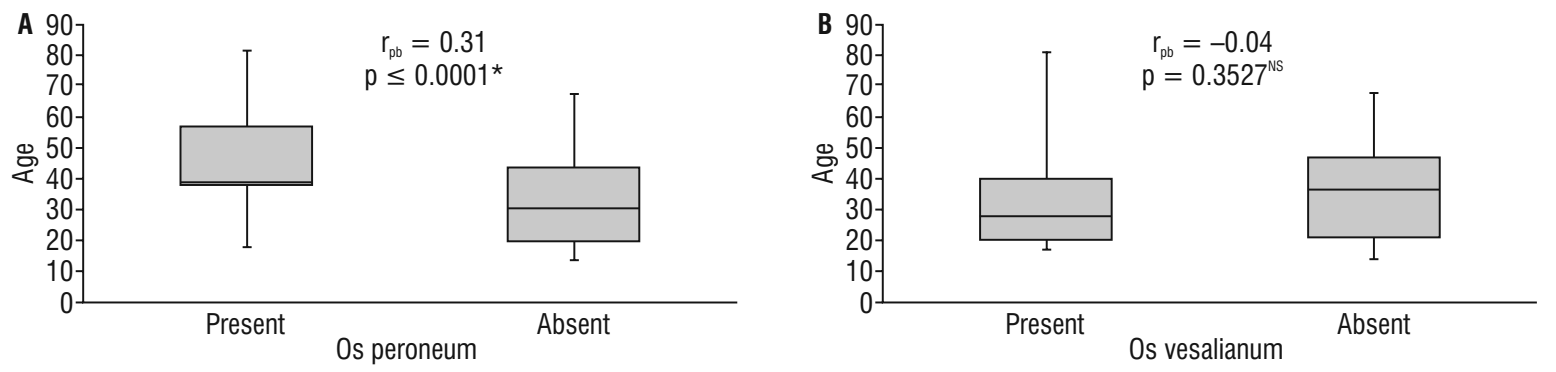

Figure 2. Box plots showing the correlation between age and presence of the lateral sesamoid bones; A. Correlation between age and os peroneum ( $p<0.0001)$; B. Correlation between age and os vesalianum $(p>0.05)$; rpb — point-biserial correlation coefficient; NS — not significant; ${ }^{*}$ significant $p<0.05$.

Table 3. The anatomical variations of the lateral foot sesamoid bones

\begin{tabular}{lc}
\hline Characteristic & N (\%) \\
\hline Number of lateral sesamoid bones & 147 \\
Os peroneum & 137 \\
Longest diameter: & \\
$\quad$ Smaller than $4 \mathrm{~mm}$ & $40(29.2 \%)$ \\
$\quad$ Between 4 and $8 \mathrm{~mm}$ & $74(54.0 \%)$ \\
$\quad$ Larger than $8 \mathrm{~mm}$ & $23(16.8 \%)$ \\
Radiographic appearance: & \\
$\quad$ Solitary & $112(81.8 \%)$ \\
$\quad$ Bipartite/multipartite & $25(18.2 \%)$ \\
Position on oblique radiographs: & \\
Close to the tubercle of the cuboid & $92(67.2 \%)$ \\
$\quad$ At the level of calcaneocuboid joint & $45(32.8 \%)$ \\
Os vesalianum & 10 \\
Type I & $4(40.0 \%)$ \\
Type II & $6(60.0 \%)$ \\
\hline
\end{tabular}

smaller than $4 \mathrm{~mm}, 54.0 \%$ (74/137) were between 4 and $8 \mathrm{~mm}$, and $16.8 \%(23 / 137)$ were larger than $8 \mathrm{~mm}$ (Fig. 3).

Regarding the location of os peroneum on oblique views, $32.8 \%(45 / 137)$ were located at the level of the calcaneocuboid joint and $67.2 \%$ (92/137) were close to the tubercle of cuboid (Fig. 3). About three fourths of os peroneum (112/137) were solitary and one fourth (25/137) were bi/multipartite (Fig. 4A, B). Only one os peroneum appeared enlarged and sclerotic, which is suggestive of a stress fracture caused by chronic overuse and repetitive activity (Fig. 4C). Among ten feet with os vesalianum, type I was identified in four cases and type II in six cases (Fig. 5).

\section{DISCUSSION}

The prevalence of the ossified form of os peroneum varies considerably in different radiographic studies $[9,10,18]$. Os peroneum in its ossified form was found in $22 \%$ of our study population. The presence of the ossified form of os peroneum was found to 

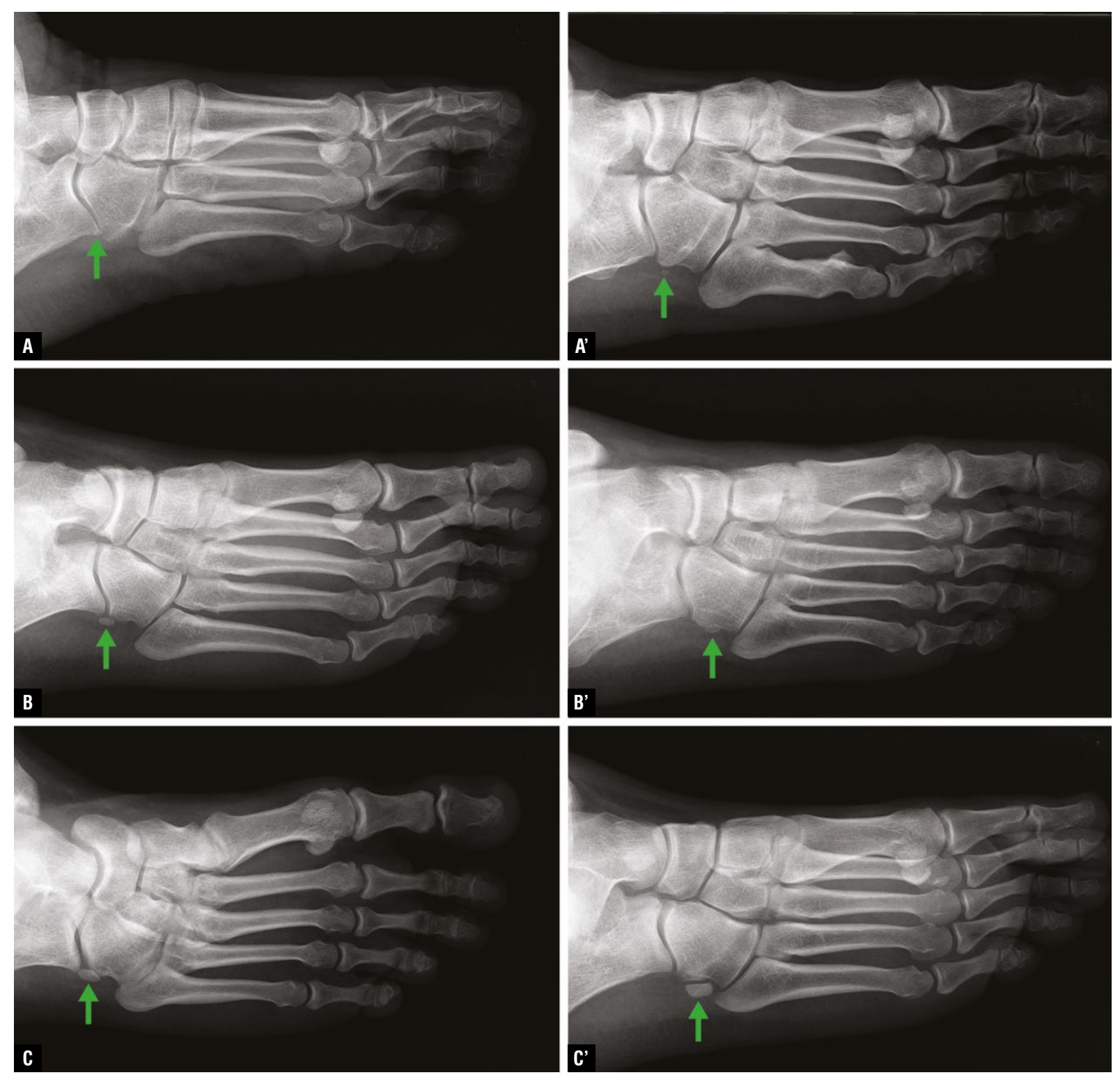

Figure 3. Radiographic anatomical variations of os peroneum according to its size and location on oblique radiographs (green arrows); A-C. Located at the level of calcaneocuboid joint; $\mathbf{A}^{\prime}-\mathbf{C}^{\prime}$. Located close to the tubercle of cuboid (distal to calcaneocuboid joint).

depend largely on age. In an anatomical cadaveric study, $30 \%$ of fibularis longus tendons obtained from a sample of 33 elderly cadavers (mean age 81 years), displayed an os peroneum both radiographically and histologically [20]. In our study, the prevalence of os peroneum rises significantly with age, and the highest prevalence was found in the elderly group $(30 \%)$. However, higher prevalence of os peroneum was reported in some anatomical studies. An anatomical study of 40 cadavers (average age: 75 years) demonstrated a $90 \%$ prevalence of os peroneum and the age has not been shown to correlate with the presence of an os peroneum [22]. Furthermore, in a study of 36 embalmed cadavers dissected bilaterally, os peroneum was found in all the tendons examined [19].

Since sesamoid bones form by endochondral ossification of sesamoid cartilages, it is anticipated that os peroneum may show varying degrees of ossification according to factors like age, genetics, mechanical loading and/or levels of physical activity $[15,20]$. It was proposed that the development of os peroneum follows various stresses and strains to the tendon, leading to its thickening and secondary ossification $[6,19]$. In our study, we were not able to include information on the patients' activity levels. 


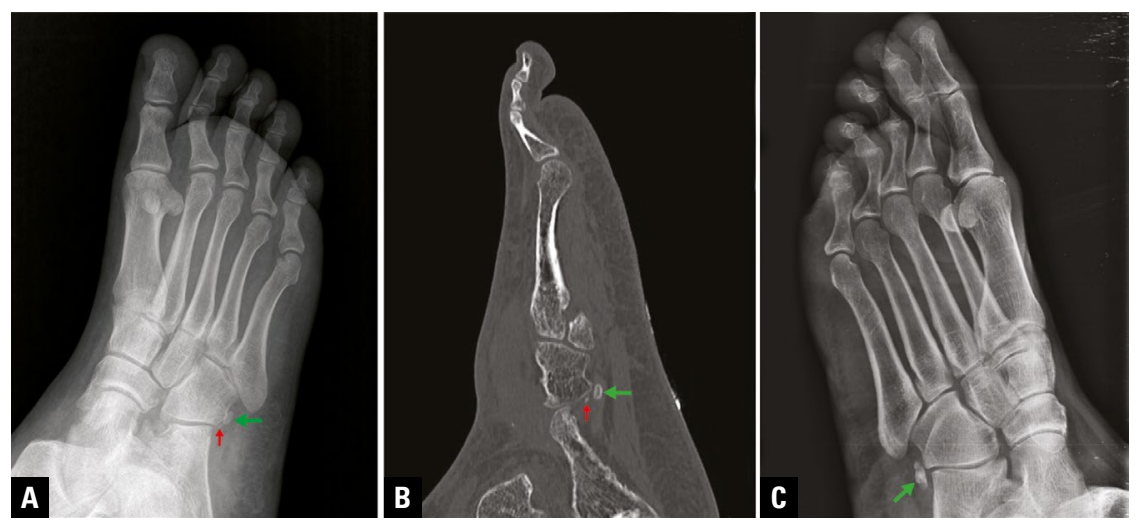

Figure 4. A. Oblique foot radiograph showing bipartite os peroneum (green and red arrows); B. Sagittal computed tomography scan of the same foot as in panel A demonstrates the bipartite os peroneum (green and red arrows); C. Oblique foot radiograph showing an enlarged and sclerotic os peroneum (green arrow).
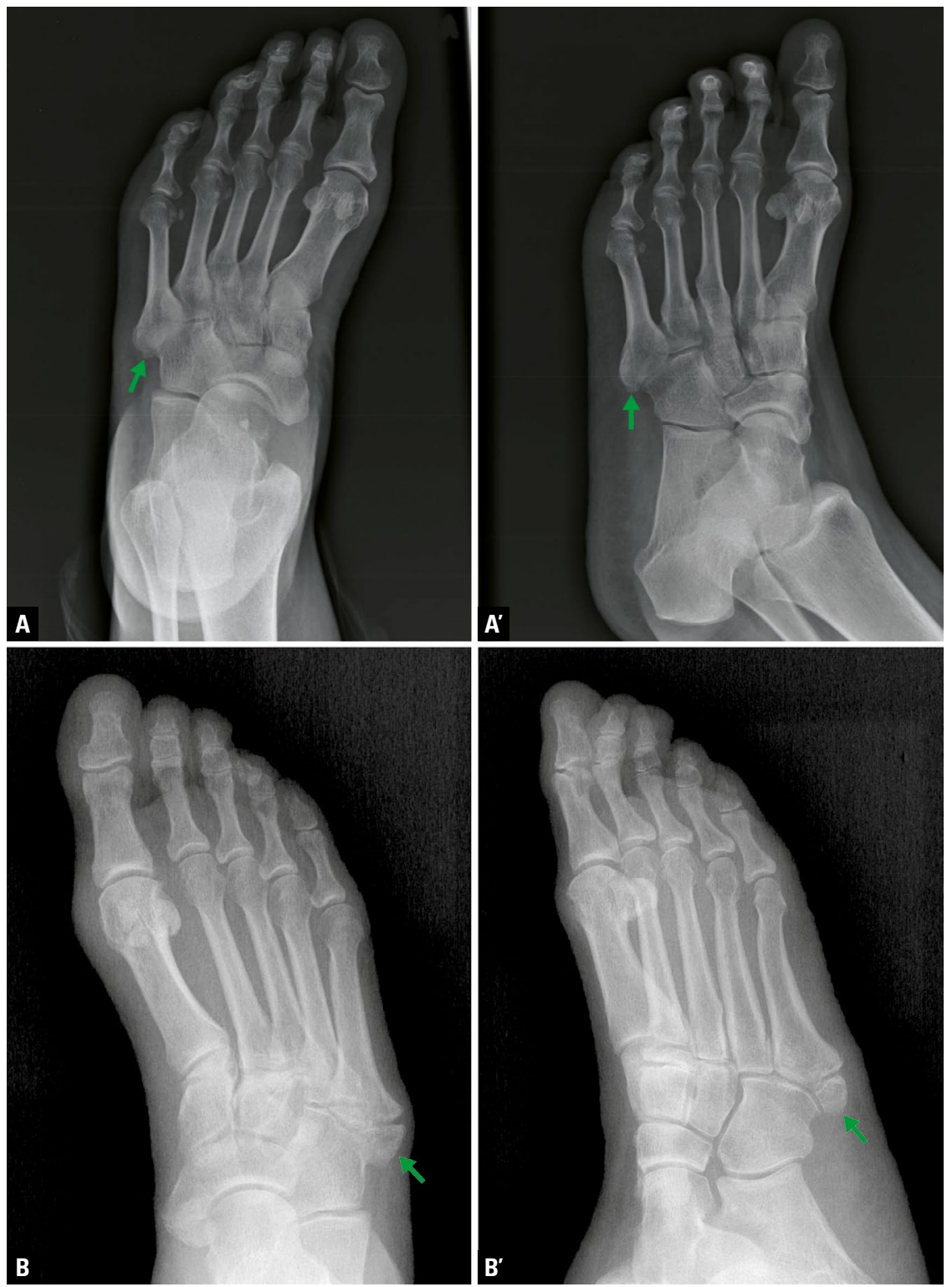

Figure 5. Radiographic anatomical variations of os vesalianum (green arrows); A, A'. Anteroposterior and oblique radiographs of type $\mathbf{l}_{\text {; }}$ B, B'. Anteroposterior and oblique radiographs of type II os vesalianum. 
Chronic lateral foot pain is a common orthopaedic foot complaint. Familiarity with various aetiologies of lateral foot pain and awareness of their typical clinical and imaging findings can prevent prolongation of undiagnosed lateral foot pain. A painful os peroneum syndrome represents a spectrum of conditions affecting the os peroneum that cause lateral foot pain. It can be acute or present as an overuse chronic condition. It includes os peroneum fracture, diastasis of a bi/multipartite os peroneum, or various fibularis longus tendon pathologies at the os peroneum ranging from tenosynovitis to tendon tear [25]. Diastasis or stress changes in bi/ multipartite os peroneum or fracture of a solitary ossicle (occur in conjunction with fibularis longus tear) are typically observed as a consequence of acute trauma [5].

Another lateral foot sesamoid bone considered in our study is os vesalianum. There has been no consensus about the exact anatomical definition of os vesalianum; it was considered to be proximal to the tip of a well-developed tuberosity of fifth metatarsal $[11,18]$. Nevertheless, os vesalianum was described by Pfitzner in 1900 as an ossicle that would constitute the tuberosity of the fifth metatarsal [27]. In our study, both descriptions were considered as types I and II, respectively. The mechanisms by which asymptomatic os vesalianum becomes symptomatic or the factors that may contribute to the development of pain are not clearly known. It was proposed that chronic inflammation caused by the instability of synchondrosis joint after repeated microtrauma or acute trauma may trigger pain [1]. Few symptomatic cases of os vesalianum have been described in the literature; most of them were described in athletes after traumatic ankle injuries $[1,7,12]$. Other reported symptomatic cases were related to repetitive microtrauma and long-lasting symptoms [14, 23]. Conversely, a symptomatic os vesalianum without a prior traumatic event or overuse was also reported [2].

\section{CONCLUSIONS}

In conclusion, different anatomical variants of the lateral sesamoid bones of the foot have been described in this study. Our data may be helpful to familiarise clinicians, who are likely to encounter a variety of clinical presentations in patients with chronic lateral foot pain, with the different morphological variants for these rare sesamoid bones.

Conflict of interest: None declared

\section{REFERENCES}

1. Aykanat F, Vincenten C, Cankus MC, et al. Lateral foot pain due to os vesalianum pedis in a young football player; a case report and review of the current literature. Skeletal Radiol. 2019; 48(11): 1821-1828, doi: 10.1007/s00256019-03190-4, indexed in Pubmed: 30815711.

2. Beil FT, Burghardt RD, Strahl A, et al. Symptomatic os vesalianum. J Am Podiatr Med Assoc. 2017; 107(2): 162-165, doi: 10.7547/15-160, indexed in Pubmed: 28394679.

3. Benjamin M, Qin S, Ralphs JR. Fibrocartilage associated with human tendons and their pulleys. J Anat. 1995; 187 (Pt 3): 625-633, indexed in Pubmed: 8586561.

4. Benjamin M, Ralphs JR. Fibrocartilage in tendons and ligaments: an adaptation to compressive load. J Anat. 1998; 193 (Pt 4): 481-494, doi: 10.1046/j.14697580.1998.19340481.x, indexed in Pubmed: 10029181.

5. Bianchi S, Bortolotto C, Draghi F. Os peroneum imaging: normal appearance and pathological findings. Insights Imaging. 2017; 8(1): 59-68, doi: 10.1007/s13244-0160540-3, indexed in Pubmed: 28058662.

6. Bizarro AH. On Sesamoid and Supernumerary Bones of the Limbs. J Anat. 1921; 55(Pt 4): 256-268, indexed in Pubmed: 17103926.

7. Boya $\mathrm{H}$, Ozcan $\mathrm{O}$, Tandoğan $\mathrm{R}$, et al. Os vesalianum pedis. J Am Podiatr Med Assoc. 2005; 95(6): 583-585, doi: 10.7547/0950583, indexed in Pubmed: 16291851.

8. Chan BY, Markhardt BK, Williams KL, et al. Os conundrum: identifying symptomatic sesamoids and accessory ossicles of the foot. Am J Roentgenol. 2019; 213(2): 417-426, doi: 10.2214/AJR.18.20761, indexed in Pubmed: 30973781.

9. Cilli $F$, Akcaoglu M. The incidence of accessory bones of the foot and their clinical significance]. Acta Orthop Traumatol Turc. 2005; 39(3): 243-246, indexed in Pubmed: 16141731

10. Coskun N, Yuksel M, Cevener M, et al. Incidence of accessory ossicles and sesamoid bones in the feet: a radiographic study of the Turkish subjects. Surg Radiol Anat. 2009; 31(1): 19-24, doi: 10.1007/s00276-008-0383-9, indexed in Pubmed: 18633564.

11. Coughlin M. Sesamoid and accessory bones of the foot. In: Coughlin M, Saltzman C, Anderson R, editors. Mann's Surgery of the Foot and Ankle 9th ed. Elsevier, Amsterdam 2013: 560-561.

12. Dorrestijn O, Brouwer RW. Bilateral symptomatic os vesalianum pedis: a case report. J Foot Ankle Surg. 2011; 50(4): 473-475, doi: 10.1053/j.jfas.2011.03.012, indexed in Pubmed: 21570323.

13. Guimerá V, Lafuente $A$, Zambrana $L$, et al. The peroneocuboid joint: morphogenesis and anatomical study. J Anat. 2015; 226(1): 104-112, doi: 10.1111/joa.12249, indexed in Pubmed: 25384452.

14. Inoue T, Yoshimura I, Ogata $K$, et al. Os vesalianum as a cause of lateral foot pain. J Pediatr Orthop B. 1999; 8(1): 56-58, doi: 10.1097/01202412-199901000-00014.

15. Le Mi. Comparative anatomy and significance of the sesamoid bone of the peroneus longus muscle (os peroneum). J Anat. 1987; 151: 85-99, indexed in Pubmed: 3654363.

16. Li X, Shi L, Liu T, et al. Progress in the clinical imaging research of bone diseases on ankle and foot sesamoid 
bones and accessory ossicles. Intractable Rare Dis Res. 2012, doi: 10.5582/irdr.2012.v1.3.122.

17. Melão L, Canella $C$, Weber $M$, et al. Ligaments of the transverse tarsal joint complex: MRI-anatomic correlation in cadavers. Am J Roentgenol. 2009; 193(3): 662-671, doi: 10.2214/AJR.08.2084, indexed in Pubmed: 19696279.

18. Mellado JM, Ramos A, Salvadó E, et al. Accessory ossicles and sesamoid bones of the ankle and foot: imaging findings, clinical significance and differential diagnosis. Eur Radiol. 2003; 13 Suppl 4: L164-L177, doi: 10.1007/ s00330-003-2011-8, indexed in Pubmed: 15018183.

19. Mittal PS, Joshi SS, Chhaparwal R, et al. Prevalence and mophometry of os peroneum amongst central Indians. J Clin Diagn Res. 2014; 8(11): AC08-AC10, doi: 10.7860/ JCDR/2014/10452.5079, indexed in Pubmed: 25584203.

20. Muehleman C, Williams J, Bareither ML. A radiologic and histologic study of the os peroneum: prevalence, morphology, and relationship to degenerative joint disease of the foot and ankle in a cadaveric sample. Clin Anat. 2009; 22(6): 747-754, doi: 10.1002/ca.20830, indexed in Pubmed: 19637293.

21. Nwawka OK, Hayashi D, Diaz LE, et al. Sesamoids and accessory ossicles of the foot: anatomical variability and related pathology. Insights Imaging. 2013; 4(5): 581-593, doi: 10.1007/s13244-013-0277-1, indexed in Pubmed: 24006205.

22. Oyedele O, Maseko C, Mkasi N, et al. High incidence of the os peroneum in a cadaver sample in Johannesburg, South Africa: possible clinical implications? Clin Anat.
2006; 19(7): 605-610, doi: 10.1002/ca.20224, indexed in Pubmed: 16287117.

23. Petrera $M$, Dwyer T, Ogilvie-Harris DJ. A rare cause of foot pain with golf swing: symptomatic os vesalianum pedis-a case report. Sports Health. 2013; 5(4): 357-359, doi: 10.1177/1941738113482446, indexed in Pubmed: 24459554

24. Sarrafian SK, Kelikian AS. Osteology. In: Kelikian AS (ed.) Sarrafian's Anatomy of the foot and ankle: Descritive, Toograhic, Functional. 3rd ed. Lippincott, Philadelphia 2011: 40-199.

25. Sobel M, Pavlov H, Geppert MJ, et al. Painful os peroneum syndrome: a spectrum of conditions responsible for plantar lateral foot pain. Foot Ankle Int. 1994; 15(3): 112-124, doi: 10.1177/107110079401500306, indexed in Pubmed: 7951939.

26. Tsuruta T, Shiokawa $Y$, Kato $A$, et al. [Radiological study of the accessory skeletal elements in the foot and ankle (author's transl)]. Nihon Seikeigeka Gakkai Zasshi. 1981; 55(4): 357-370, indexed in Pubmed: 7276670.

27. Vaz A, Trippia CR. Small but troublesome: accessory ossicles with clinical significance. Radiol Bras. 2018; 51(4): 248-256, doi: 10.1590/0100-3984.2017.0147, indexed in Pubmed: 30202129.

28. Wang XT, Rosenberg ZS, Mechlin MB, et al. Normal variants and diseases of the peroneal tendons and superior peroneal retinaculum: MR imaging features. Radiographics. 2005; 25(3): 587-602, doi: 10.1148/rg.253045123, indexed in Pubmed: 15888611. 